\title{
Maximum entropy principle in recurrence plot analysis on stochastic and chaotic systems
}

Cite as: Chaos 30, 043123 (2020); https://doi.org/10.1063/1.5125921

Submitted: 27 August 2019 . Accepted: 27 March 2020 . Published Online: 17 April 2020

T. L. Prado (D), G. Corso (D, G. Z. dos Santos Lima (D), R. C. Budzinski (D, B. R. R. Boaretto (D) F. A. S. Ferrari, E. E. N. Macau (D), and S. R. Lopes (D)

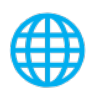

\section{ARTICLES YOU MAY BE INTERESTED IN}

Strong correlations between power-law growth of COVID-19 in four continents and the inefficiency of soft quarantine strategies

Chaos: An Interdisciplinary Journal of Nonlinear Science 30, 041102 (2020); https:// doi.org/10.1063/5.0009454

\section{A network model for polarization of political opinion}

Chaos: An Interdisciplinary Journal of Nonlinear Science 30, 043109 (2020); https:// doi.org/10.1063/1.5131018

Nonstationary coherence-incoherence patterns in nonlocally coupled heterogeneous phase oscillators

Chaos: An Interdisciplinary Journal of Nonlinear Science 30, 043103 (2020); https:// doi.org/10.1063/1.5145259

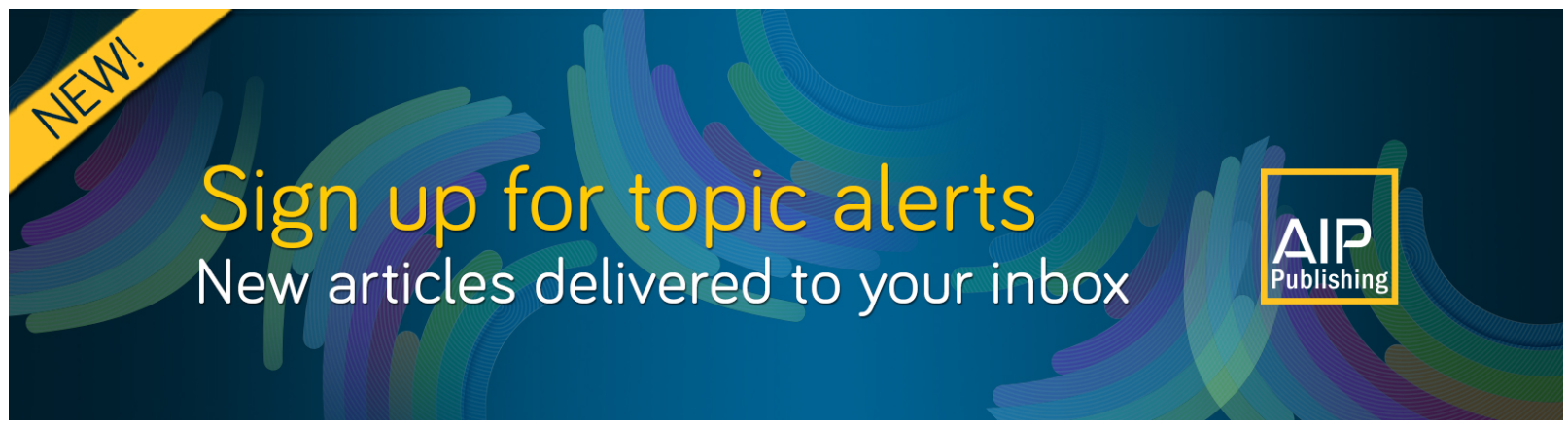




\title{
Maximum entropy principle in recurrence plot analysis on stochastic and chaotic systems
}

Cite as: Chaos 30, 043123 (2020); doi: 10.1063/1.5125921

Submitted: 27 August 2019 . Accepted: 27 March 2020 .

Published Online: 17 April 2020

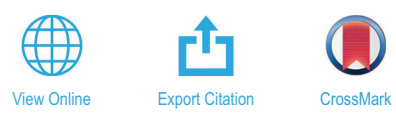

T. L. Prado, , , a) (D) G. Corso, ${ }^{2}$ (D) G. Z. dos Santos Lima, ${ }^{2,3}$ (D) R. C. Budzinski, ${ }^{1}$ (D) B. R. R. Boaretto, ' (D) F. A. S. Ferrari, E. E. N. Macau, 5,6 (D) and S. R. Lopes ${ }^{7, b)}$ (i)

\begin{abstract}
AFFILIATIONS
${ }^{1}$ Departamento de Física, Universidade Federal do Paraná, Curitiba 81531-980, Brazil

${ }^{2}$ Departamento de Biofísica e Farmacologia, Universidade Federal do Rio Grande do Norte, Natal 59078-970, Brazil

${ }^{3}$ Escola de Ciências e Tecnologia, Universidade Federal do Rio Grande do Norte, Natal 59078-970, Brazil

${ }^{4}$ Instituto de Engenharia, Ciência e Tecnologia, Universidade Federal dos Vales do Jequitinhonha e Mucuri, Janaúba 39447-790, Brazil

${ }^{5}$ Laboratório Associado de Computação e Matemática Aplicada, Instituto Nacional de Pesquisas Espaciais, São José dos Campos 12227-010, Brazil

${ }^{6}$ Instituto de Ciência e Tecnologia, Universidade Federal de São Paulo, São Jose Dos Campos 12231-280, Brazil
\end{abstract}

\author{
a) Author to whom correspondence should be addressed: thiago@fisica.ufpr.br \\ ${ }^{\text {b) }}$ Electronic mail: lopes@fisica.ufpr.br
}

\begin{abstract}
The recurrence analysis of dynamic systems has been studied since Poincaré's seminal work. Since then, several approaches have been developed to study recurrence properties in nonlinear dynamical systems. In this work, we study the recently developed entropy of recurrence microstates. We propose a new quantifier, the maximum entropy $\left(S_{\max }\right)$. The new concept uses the diversity of microstates of the recurrence plot and is able to set automatically the optimum recurrence neighborhood ( $\epsilon$-vicinity), turning the analysis free of the vicinity parameter. In addition, $\epsilon$ turns out to be a novel quantifier of dynamical properties itself. We apply $S_{\max }$ and the optimum $\epsilon$ to deterministic and stochastic systems. The $S_{\max }$ quantifier has a higher correlation with the Lyapunov exponent and, since it is a parameter-free measure, a more useful recurrence quantifier of time series.
\end{abstract}

Published under license by AIP Publishing. https://doi.org/10.1063/1.5125921

The entropy of recurrence microstates evaluates the underlying complex features of dynamical trajectories. A relevant issue to achieve adequate results in recurrence analysis is the vicinity threshold choice, in general, a free parameter in recurrence analyses. In this work, we propose that the maximum entropy principle allows us to set a unique entropy and also an optimum vicinity threshold value. This uniqueness turns the vicinity threshold parameter into a new recurrence quantifier. Furthermore, the maximum entropy principle turns recurrence analysis a parameter-free tool. This approach is applied to chaotic and stochastic data, and the results improve substantially compared with the previous recurrence analysis making use of other vicinity threshold choices.

\section{INTRODUCTION}

The maximum entropy principle has a long history in science where it is used in several frameworks such as statistical physics, ${ }^{1,2}$ ecology, ${ }^{3}$ neuroscience, ${ }^{4}$ and thermodynamics. ${ }^{5}$ Moreover, the Shannon information theory explores the maximization of entropy to construct the modern science of computation. ${ }^{6}$ On the other hand, the likelihood concept on statistics ${ }^{7}$ is an alternative view of the entropy optimization. The entropy function itself is widely used as a complexity index or an index to characterize the diversity of elements in a system. The use of entropy as an index is common in biodiversity and ecology, ${ }^{8}$ acoustic environments, ${ }^{9}$ linguistic elements in a text, ${ }^{10}$ and time series. 
The theory of the recurrence analysis connects to the Poincaré concept ${ }^{12}$ where dynamical trajectories return to a small region of the phase space defined by a vicinity of size $\epsilon$. The Recurrence Plot (RP) method can be used to obtain visual insights about the underlying dynamics, while a more quantitative view can be obtained through recurrence quantification analyses (RQAs), ${ }^{13,14}$ which quantify structures and properties of the RP. We call attention to the use of entropy in the recurrence analysis in dynamical systems ${ }^{15}$ and the maximum entropy concept in symbolic recurrence analysis. ${ }^{16}$ Recently, a recurrence quantifier named recurrence entropy has been developed. ${ }^{17}$ This new recurrence quantifier constructs an entropy over the diversity of microstates of the Recurrence Plot (RP).

The RP and the corresponding RQAs are highly sensitive to the choice of $\epsilon$ and exhibit poor performance for non-optimal choices. The vicinity $\epsilon$ is the major free parameter of the RP methodology. Previous works have suggested different approaches to choose a suitable $\epsilon,{ }^{14,18-20}$ although an optimum choice remains an open research topic.

In the present work, we connect the recurrence entropy developed in Ref. 17 with the maximum entropy principle. ${ }^{1}$ We propose a novel approach to quantify RPs, which is constructed using maximization of the recurrence entropy $\left(\mathrm{S}_{\max }\right)$ by adjusting the vicinity $\epsilon$. The main advantage of the method is that the definition of $S_{\max }$ does not depend on free parameters. Additionally, in this new context, the vicinity $\epsilon$ becomes a new RQA. The use of the entropy based on recurrence microstates was presented as an appropriate approach in Ref. 17. Here, we show that the maximum of the recurrence entropy provides better recurrence analysis results and automatically sets an optimal vicinity parameter for a recurrence quantification analysis.

The paper is organized as follows: Sec. II presents the traditional recurrence plot, the recurrence quantification analysis methods, the recently developed entropy of recurrence microstates, and the maximum recurrence entropy. Section III contains the results of the maximum entropy principle applied to chaotic and stochastic data, while in Sec. IV, we outline our conclusions.

\section{METHODOLOGY}

This section is divided into three parts. First, we introduce the recurrence plot technique. Then, we discuss the recently introduced entropy based on the recurrence microstates, and after that, we apply the maximum entropy principle to the recurrence plot structure. This approach reduces the number of free parameters by introducing a single concept while increases the reliability and capacity of analysis of the methodology.

\section{A. Recurrence plots and recurrence quantification analysis}

The concept of recurrence dates back to Henri Poincaré, ${ }^{12}$ who considered it a fundamental feature of dynamical systems. A modern visualization method known as a recurrence plot (RP) introduced in Ref. 21 is based on the recurrence matrix $\mathbf{R}_{i j}$ defined as

$$
\mathbf{R}_{i j}(\epsilon)=\Theta\left(\epsilon-\left|\mathbf{x}_{i}-\mathbf{x}_{j}\right|\right), \mathbf{x}_{i} \in \mathbb{R}, \quad i, j=1,2, \ldots, K,
$$

where $\mathbf{x}_{i}$ and $\mathbf{x}_{j}$ represent dynamical states at time $i$ and $j, \Theta$ is the Heaviside function, $K$ is the length of the analyzed time series interval, and $\epsilon$ is the threshold or vicinity parameter. The parameter $\epsilon$ consists of the maximum distance between two points in a trajectory in the phase space, such that both points can be considered recurrent to each other. We note that $\mathbf{R}_{i j}(\epsilon)$ is an explicit function of the threshold distance $\epsilon$. Moreover, the RP is a symmetric matrix of "ones" and "zeros" where a one (zero) intends for recurrent (non-recurrent) points in the phase space.

In this way, the recurrence plot is a graphical binary representation of the recurrence patterns extracted from the time series ${ }^{21}$ or spatial profiles. ${ }^{22,23}$ It was developed by Eckmann et al. ${ }^{21}$ and further explored by several authors. A good compilation in the literature on this issue is found in Marwan et al. ${ }^{14}$ Important characteristics of recurrence plots are the presence of finite length diagonal lines, indicating periodic signals or recurrence segments and isolated points, suggesting stochastic and/or chaotic signals. Moreover, specific features of a time series can be obtained by using a set of tools developed by Zbilut and Webber, Jr., ${ }^{13,24}$ who measure the signal complexity based on the recurrence matrix. This process and the extracted features are called recurrence quantification analysis (RQA) or recurrence quantifiers.

The RQA identifies different aspects of the recurrence plot from the density of recurrent points to the statistics of vertical (horizontal) or diagonal lines. ${ }^{14}$ To avoid problems with very large recurrence plots, when analyzing long time series, it is convenient to divide the original time series into smaller sub-series (or windows) of length $K$ such that $K \ll M$, where $M$ is the length of the entire trajectory. For each window, we construct a recurrence matrix that is used to compute the recurrence quantifiers. The simplest RQA is the recurrence rate (RR) defined as the density of recurrent points in $\mathbf{R}_{i j}$. Others, such as laminarity or trapping time, evaluate vertical line distributions, which are connected with dynamical intermittency. Diagonal lines represent recurrence segments of trajectories, for which there are several quantifiers that evaluate the diagonal line structure, such as determinism or divergence. Two pieces of a trajectory following a diagonal line undergo a similar evolution, once they have visited the same region of the phase space at different times. This is the key idea behind recurrence and thus a clear-cut signature of a deterministic behavior in the time series.

\section{B. The entropy of recurrence microstates}

To properly define entropy, we use the concept of microstates for a RP, an idea that is associated with features of the time series dynamics. These microstates are evaluated using small matrices of dimension $N \times N$ that are sampled from the RP. The matrices can assume several configurations as can be seen in Fig. 1 for the particular situation $N=2$. The total number of microstates for a given $N$ is $N^{*}=2^{N^{2}}$

To associate probabilities to the microstates, we sample $\bar{N}$ random matrices $N \times N$ from the RP. We call $n_{i}$ the number of observed matrices in a particular microstate $i$ in a sample of $\bar{N}$ elements; by definition, we have $\bar{N}=\sum_{i=1}^{N^{*}} n_{i}$. In this way, the quantity $P_{i}=n_{i} / \bar{N}$ is the probability of microstate $i$. We define the entropy of the RP 
associated with the probabilities of the set of microstates as

$$
\mathrm{S}=-\sum_{i=1}^{i=N^{*}} P_{i} \ln P_{i}
$$

this methodology was developed by Corso et al. ${ }^{17}$ The parameters $N$ and $N_{b a r}$ are not properly free parameters, since in Marwan et al., ${ }^{14}$ the authors present a guide for a constant $\epsilon$, which also applies for a maximum entropy choice.

In Fig. 1, we depict all possible microstates for $N=2$. They were split into groups according to the number of recurrences in each microstate. In this figure, we also show a binary representation of the $N^{*}$ microstates using four bits. This symbolic sequence is used in the algorithm that samples, identifies, and counts microstates from the RP.

Figure 2 presents how microstates are computed from a schematic data series. The figure illustrates diagonal and verti$\mathrm{cal} /$ horizontal structures associated with microstates. Similar examples can be developed for each distinct microstate and larger values of $N$.

The recurrence matrix is the line of identity (LOI) symmetric. Each microstate is extracted from one or the other side of the triangular recurrence matrix to avoid the overestimation of some microstates. Figure 3 presents some microstate structures that can

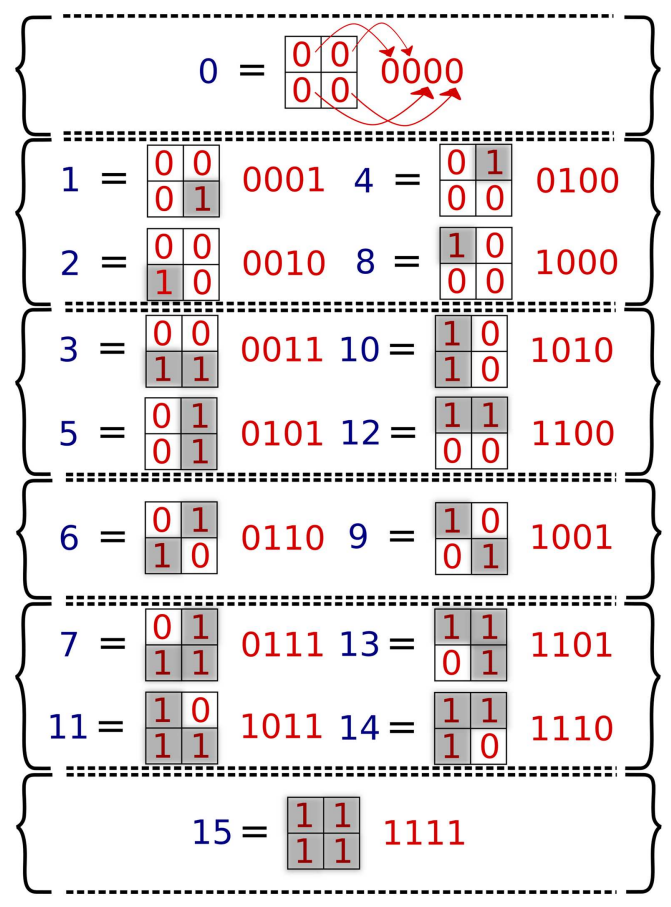

FIG. 1. Representation of microstates. This figure shows the actual microstates for a sample matrix of size $N=2$, along with the correspondent binary code or symbolic representation. In this current configuration, there are 16 different combinations of microstates that can be grouped according to the number of recurrent points. In this example, we identify six classes of microstates that correspond to the number of recurrent elements using the matrix [2×2].

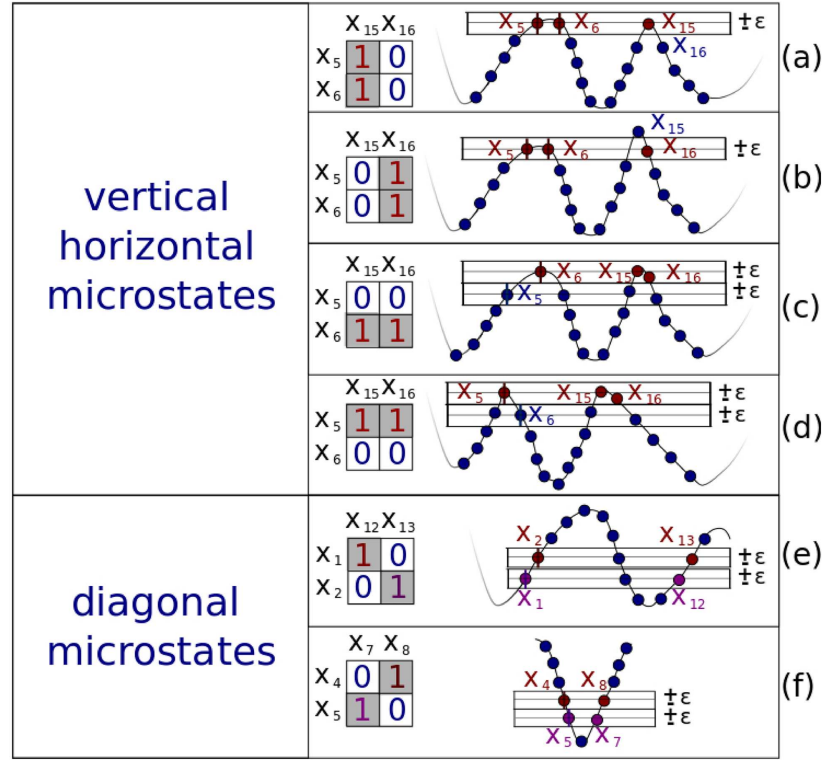

FIG. 2. Dynamical microstate interpretation of the time series. Here, we show $N=2$ examples of microstates for the schematic time series shown. Differently from Fig. 1, we depict dynamical possible temporal (or spatial) patterns to the case of $N=2$ for vertical (a) and (b), horizontal (c) and (d), and diagonal (e) and (f) microstates.

change from extraction from one or the other side of the LOI. These microstates that contain the same dynamical information but different microstate structures are called degenerate microstates.

\section{The maximum entropy of recurrence microstates}

The recurrence plot technique and corresponding recurrence quantification methods depend directly on the choice of $\epsilon$. This is a known feature of recurrence analysis and can bias the results. The

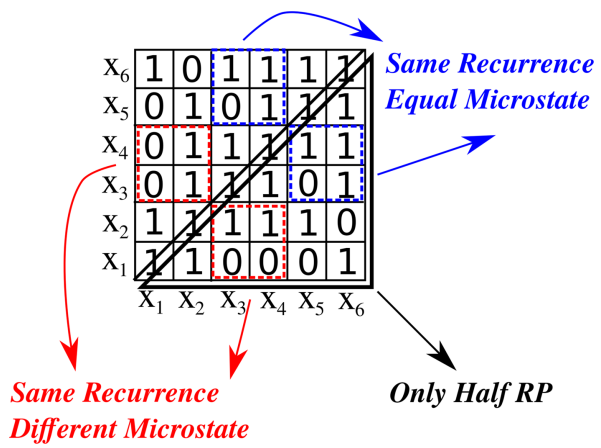

FIG. 3. Detail from overestimation of distinct microstates. A schematic recurrence matrix presenting two dynamical segment recurrences and the associated microstate from both sides of LOI. Some microstates depict degenerescence when evaluated for both sides of LOI. 


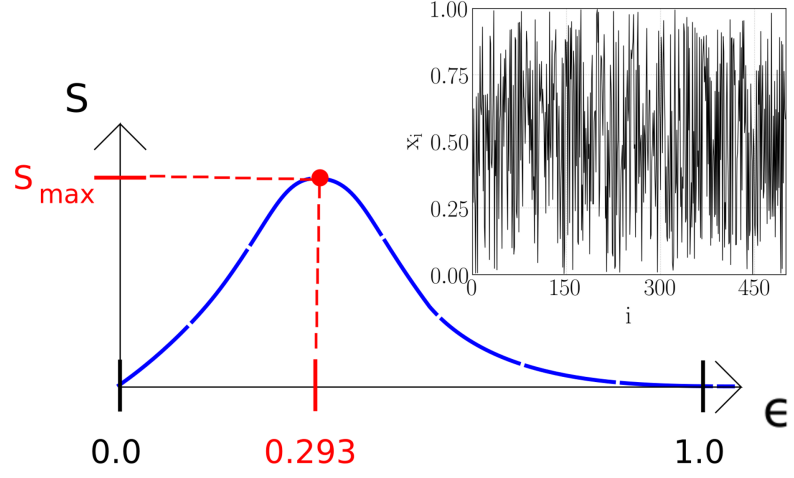

FIG. 4. Entropy as a function of the vicinity threshold. The curve of the entropy vs the vicinity epsilon $(\epsilon)$ typically shows a maximum value, which corresponds to the maximum entropy. In this particular example, we take the white noise time series (panel inset) and vary $\epsilon$ to find the maximum entropy $\left(S_{\max }\right)$. In this simulation, we choose $N=2$ (see Fig. 2)

entropy of recurrence microstates (S) also suffers from this issue. ${ }^{17}$ Here, we propose a method to select an optimal choice of $\epsilon$, which leads to a new quantifier.

Several approaches to select the threshold parameter $\epsilon$ have been proposed in the literature, some of them involve the standard deviation as proposed by Thiel et al., ${ }^{18}$ while others propose some percentage of a maximum phase space diameter, ${ }^{25}$ typically not exceeding $10 \%{ }^{24}$ We advocate the concept of maximization of the microstate entropy, even if data are periodic, stochastic, experimental, or simulated.

Consider the representation of $\mathrm{S}$ in Fig. 4 as a function of $\epsilon$. In this case, we observe that $S(\epsilon)$ has a well-defined extreme. The point of the maximum will change according to the analyzed system. For the white noise, which has a uniform distribution of values in the domain, it is possible to deduce the threshold by geometrical arguments, and the RR quantifier is $\epsilon \approx 0.293$; see Ref. 17 . The maximum value of entropy is computationally estimated for each system and depends on its dynamical characteristics. We have used the divide-and-conquer algorithm to find the maximum microstate entropy.

The choice on the maximum Shannon entropy takes into account the important feature of no possibility exclusion. ${ }^{1}$ Considering the Shannon entropy in recurrences, the most important feature in favor to set the system maximal entropy is that this choice eliminates the dependencies of recurrence plot techniques on free parameters. $^{20}$

\section{EXPLORING THE METHODOLOGY ON DYNAMICAL MODELS}

In this section, we present the maximum entropy concept applied to chaotic and stochastic systems. This section is divided as follows: (A) the analysis of the logistic map, a paradigmatic chaotic map, and (B) the analysis of stochastic maps: the white and Gaussian noises.

\section{A. Study of the logistic map}

The logistic map is a well known complex dynamical map with a periodic regime, a transition to chaos and regions of periodic windows entangled with chaotic behavior. The iterative equation of the logistic map is

$$
x_{n+1}=x_{n} r\left(1-x_{n}\right), \quad 0 \leq x_{n} \leq 1,
$$

where the chaotic region with windows of periodicity is achieved by varying the parameter $r$ in the interval $3.6<r<4.0$.

The complexity involving the recurrence entropy $S$, the vicinity $\epsilon$, and the Lyapunov exponent $\lambda$ in the logistic map are illustrated in Fig. 5. In panel (a), we show $S$ with the help of a coding color; in the horizontal axis, we plot $\epsilon$ and in the vertical axis, the parameter $r$ of the logistic map. Correspondingly, panel (b) displays $\lambda$ for the same $r$ interval. We notice in Figs. 5(a) and 5(b) the presence of periodic windows of the logistic map $(\lambda<0)$ with a clear decrease in S. In panel (a), we also indicate, with black dots, the maximal value of entropy $S_{\max }$ relative to optimal $\epsilon$. In Fig. 6, we explore in more detail the correspondence between $S_{\max }$ and the Lyapunov exponent $\lambda$.

In Fig. 6(a), we present the behavior of the recurrence entropy vs $r$ for the logistic map. As shown in Ref. 17, the increase in S follows chaotic (periodic) regions, as presented in Fig. 6. Although both $S$ curves follow the increase on $r$, in periodic windows, the dynamical bifurcations are more clearly evaluated using $S_{\max }$, as observed in the inset of Fig. 6(a). For comparison, Fig. 6(b) displays the Lyapunov exponent $\lambda$ against the nonlinear parameter $r$. We observe a strong correlation between $\lambda$ and $S_{\max }$. In fact, in Fig. 7, we explore in more detail this relation.

In order to test the recurrence entropy as an index of complexity, we compare $S$ and $S_{\max }$ against the Lyapunov exponent using data from the logistic map. For the test, we employ $3.6<r<4.0$ to guarantee the logistic map inside a region with chaotic regimes entangled with periodic window regimes. Figure 7 shows the Lyapunov coefficient vs the entropy: for (a) $S(\epsilon=0.1)$, (b) $S(\epsilon=0.2$ ), and $(c) \epsilon$ free to attain the maximal entropy $\left(S_{\max }\right)$. Panel $(d)$ presents the Pearson correlation coefficient between $\lambda$ and $S(\epsilon)$, and the correlation coefficient is plotted as a function of $\epsilon$. The dashed line in the plot corresponds to the reference line with the correlation between the Lyapunov exponent and the maximum recurrence entropy. The Pearson correlation coefficient curve vs $\epsilon$ reveals a large $\epsilon$ interval with a high correlation with the Lyapunov exponent; however, Fig. 7 (d) makes clear that the correlation of $S_{\max }$ is always higher than any correlation of entropy with fixed $\epsilon$. For additional comparison, consider Table I in which we show the Pearson correlation coefficient between the Lyapunov exponent and usual recurrence quantifiers.

The simulations were performed with a time window of size $\mathrm{K}=1 \times 10^{3}$, after an appropriate transient time, and $(l, v)_{\min }=2$ (minimum diagonal/vertical lines). For further reading on recurrence quantification methods, see Refs. 14 and 27. We note that $S_{\max }$ shows a higher (anti)correlation when compared to DIV (Divergence) and DET (Determinism), both quantifiers connected to diagonal recurrent structures; LAM (Laminarity-vertical/horizontal recurrent structures); ENTR (Entropy of recurrent diagonal lines); and $S_{r t}$ (Recurrence time entropy) for several different $\epsilon$ choice 


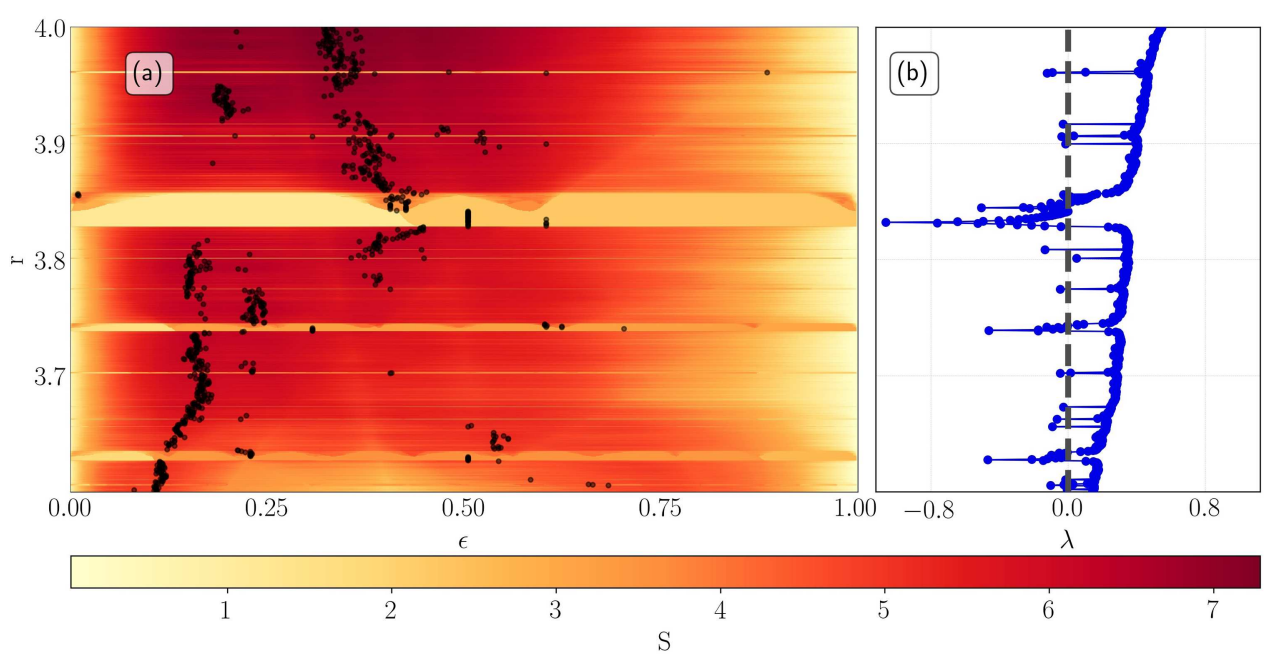

FIG. 5. Recurrence entropy and the Lyapunov exponent for the logistic map. Panel (a) presents the recurrence entropy using code colors; the vertical axis is the logistic parameter $r$ and in the horizontal axis, we show the vicinity $\epsilon$. The dots mark the maximum entropy values. In (b), we show the Lyapunov exponent for the same $r$ interval.

approaches. Particularly, DIV and $\mathrm{S}_{\mathrm{rt}}$ show a good correlation with the highest Lyapunov exponent, but the correlation is indeed lower than for $S_{\max }$. Furthermore, the changes on $\epsilon$ due to the $S$ maximization turn $\epsilon$ into another important recurrence quantifier, which may be further explored in stochastic data.

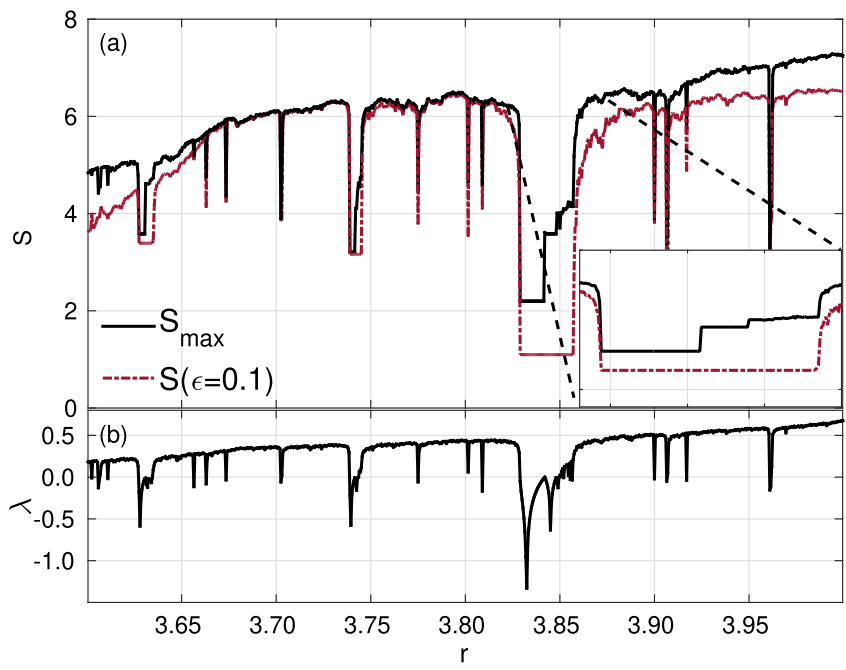

FIG. 6. Logistic map: entropy and the Lyapunov exponent. (a) displays two curves, $\mathrm{S}_{\max }$ (solid line) and $\mathrm{S}(\epsilon=0.1)$ (dashed line), while (b) presents the Lyapunov exponent. The curves analyze the logistic map for $3.6 \leq r \leq 4.0$. Comparing (a) and (b), we observe that both entropy curves approximately follow $\lambda$, although the correlation in periodic windows and the increase rate is better represented by $S_{\max }$. In the computation of the $S$ curves, we used 10 samples (error bars suppressed due to a small deviation), $N=4, \bar{N}=10^{5}$, and $K=1000$.

\section{B. Stochasticity and maximum recurrence entropy 1. White noise}

We start our analysis of stochastic signals with the white noise. We construct the white noise, $y_{W}(t)$, with random data from a
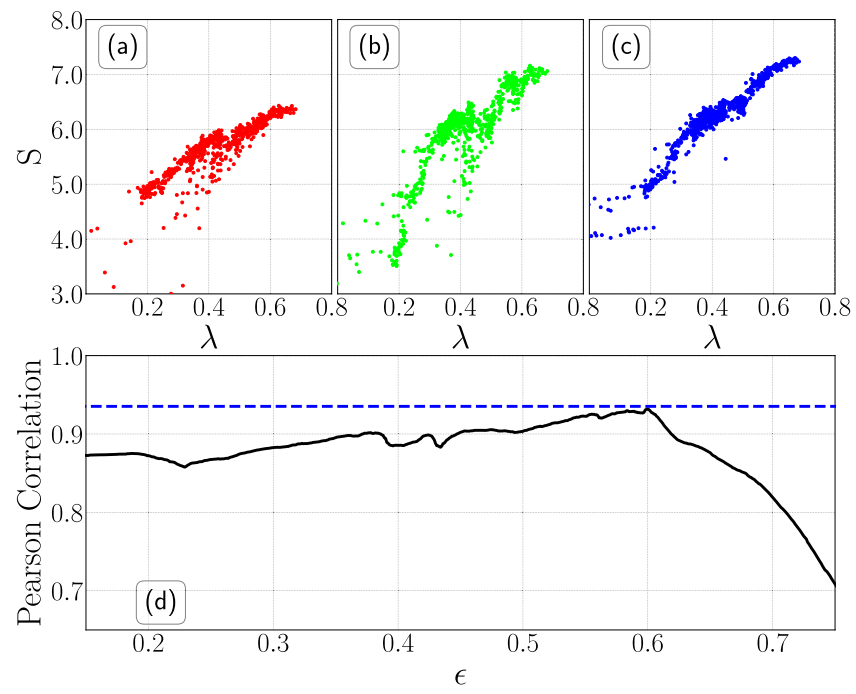

FIG. 7. Correlation between recurrence entropy and the Lyapunov exponent $\lambda$. The logistic map for $3.6 \leq r \leq 4.0$ was considered in (a)-(d). Figures (a)-(c) show the Lyapunov exponent against $\mathrm{S}$. A fixed $\epsilon=0.1$ was used in (a) and $\epsilon=0.2$ in (b) and in (c) a variable $\epsilon$ that produces the maximal entropy. In (d), we present the curve of $S$ for fixed $\epsilon$ (solid line) and the reference line of $S_{\max }$ (dashed line) for variable $\epsilon$. All curves and the reference line were done with 10 samples (error bars have been suppressed due to a small deviation), $N=4, \bar{N}=10^{5}$, and $K=1000$ 
TABLE I. Mean Pearson correlation coefficient between the Lyapunov exponent and the recurrence quantifiers: DIV, DET, LAM, ENTR, and $\mathrm{S}_{\mathrm{rt}}$ for different $\epsilon$ choices $[\epsilon(\mathrm{RR})$ is for fixed RR-Recurrence Rate-quantifier]. The employed dataset is a time series from the logistic map. The Pearson coefficient relative to the maximal entropy $\mathrm{S}(\mathrm{N}=4)_{\max }$ is approximately 0.94 . The standard deviation of table coefficients is lower than $10^{-2}$

\begin{tabular}{lccccc}
\hline \hline & \multicolumn{5}{c}{$\mathrm{S}(\mathrm{N}=4)_{\max } \approx 0.94$} \\
\cline { 2 - 6 } & DIV & DET & LAM & ENTR & $\mathrm{S}_{\mathrm{rt}}$ \\
\hline$\epsilon=0.1$ & 0.86 & -0.69 & 0.35 & -0.76 & 0.87 \\
$\epsilon(\mathrm{RR}=0.1)$ & 0.87 & -0.68 & 0.27 & -0.65 & 0.89 \\
$\epsilon(\mathrm{RR}=0.2)$ & 0.87 & -0.28 & 0.38 & -0.64 & 0.86 \\
$\epsilon(\mathrm{RR}=0.5)$ & 0.86 & 0.49 & 0.07 & -0.48 & 0.70 \\
\hline \hline
\end{tabular}

uniform distribution; for simplicity, we employ a random generator with values between 0 and 1 . We construct a large time series $y_{W}(t)$ with $0<t<10^{5}$. To compute $\mathrm{S}_{\max }$ and $\epsilon$, we use moving windows of size $K=1000$; in addition, we use $\bar{N}=2 \times 10^{4}$ for $N=2,3$ and $\bar{N}=10^{5}$ for $N=4$. Higher values for $N$ typically demand higher sampling to have a good resolution. The simulation estimated values of the maximal entropy for the white noise are $S_{\max }^{W}(N=2)$ $=2.692 \pm 0.001, S_{\max }^{W}(N=3)=5.737 \pm 0.004$, and $S_{\max }^{W}(N=4)$ $=9.357 \pm 0.007$; the corresponding vicinity sizes to attain the maximal entropy are $\epsilon(N=2)=0.281 \pm 0.001, \epsilon(N=3)=0.266 \pm$ 0.001 , and $\epsilon(N=4)=0.249 \pm 0.002$.

The theoretical value of $S_{\max }$ is computed assuming the equiprobability of all microstates. As the number of microstates is calculated by $N^{*}=2^{N^{2}}$, the maximal entropy is

$$
S_{\max }^{\text {theory }}=\ln \left(N^{*}\right)=N^{2} \ln (2) \text {. }
$$

Using this equation, we compute $S_{\max }^{\text {theory }}(N=2)=2.772$, $S_{\max }^{\text {theory }}(N=3)=6.238$, and $S_{\max }^{\text {theory }}(N=4)=11.09$. The predicted theoretical estimations are quite close to the computational values for the cases $N=2,3$, and $4 ; S_{\max }^{\text {theory }} \sim S_{\max }^{W}$. Indeed, the values of $S_{\max }^{\text {theory }}$ are slightly higher than $S_{\max }^{W}$ because the theory is an upper bond estimation based on a hypothetical equiprobability among the microstates.

In the work, ${ }^{17}$ a model is developed based on the assumption of maximal entropy for $R R=0.5$ and a theoretical value for the vicinity size $\epsilon_{\max }^{\text {theory }} \approx 0.293$ is derived. This value is close to the computational value estimated for $N=2,3$, and $4 ; \epsilon_{\max }^{\text {theory }} \sim \epsilon_{\max }^{W}$, with increased distinction for higher values of $N$.

\section{Gaussian noise}

The Gaussian noise is constructed using a set of $\eta$ similar white noise series with different seeds in the pseudo-random generator. For a white noise $y_{W}(t)$ with $1 \leq t \leq K$, we define the following series:

$$
y_{G}^{\eta}(t)=\frac{1}{\eta} \sum_{i=1}^{\eta} y_{W}(t) .
$$

In the limit of small $\eta$, the series $y_{G}^{\eta}(t)$ behaves like the usual white noise, and true Gaussian noise is formed in the limit of large $\eta$. In

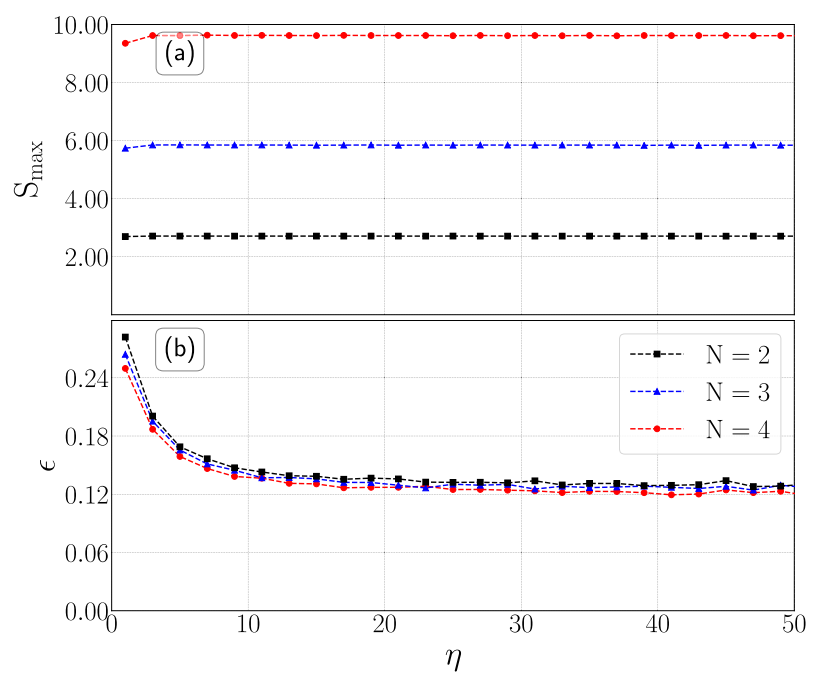

FIG. 8. Maximum entropy analysis of Gaussian noise. Each panel contains three curves corresponding to microstate sizes $N=2,3$, and 4 , in black (dashed-squared), blue (dashed-triangle), and red (dashed-circle) colors. Panel (a) presents the maximum entropy as a function of $\eta$ ( $\eta=$ the number of white noise time series added up). Note that the maximum entropy values are similar to the white noise results. In panel (b), we observe the changes in $\epsilon$ respective to the maximum entropy.

Fig. 8, we illustrate the analysis of the recurrence entropy of the Gaussian noise for several $\eta$; for the construction of these figures, 20 simulations were performed. In addition, for each figure, we used $K=1000$ and $\bar{N}=2 \times 10^{4}$ for $N=2,3$ but $\bar{N}=10^{5}$ for $N=4$.

An analysis of Fig. 8(a) reveals that estimated values of the maximal entropy are $S_{\max }^{G}(N=2)=2.707 \pm 0.001, S_{\max }^{G}(N=3)$ $=5.841 \pm 0.003$, and $S_{\max }^{G}(N=4)=9.616 \pm 0.005$. As expected, the entropy shows the similar values of the white noise and fit with the theoretical predictions (4). The values of $S_{\max }$ are independent of $\eta$, but this is not true for $\epsilon$ [Fig. 8(b)]. For small $\eta$, the time series behaves like the white noise and assumes the same $\epsilon \approx 0.293$, but for large $\eta$, the computed $\epsilon$ decreases to an asymptotic value of $\epsilon \approx 0.125$. Both are approximately the standard deviation of the respective analyzed data.

The parameter $\eta$ controls the transition from white noise to Gaussian noise. The white noise show a uniform distribution. The Gaussian noise in comparison has a centralized distribution, which reduces the necessity of larger $\epsilon$ for a large amount of recurrent states. The reduction of $\epsilon$ for the transition from white to Gaussian noise is presented clearly in Fig. 8(b).

\section{CONCLUSION}

In this paper, we present a new approach based on the maximum entropy principle for the recurrence microstates. We tested the method for both stochastic and chaotic data. The most evident advantages of our approach are (i) the automatic $\epsilon$ choice defined by entropy maximization, (ii) the possibility of this optimal choice of $\epsilon$ be used in recurrence quantification analyses, ${ }^{14}$ and 
(iii) a good agreement for $\mathrm{S}_{\max }$ between theoretical and simulated results. In addition, the maximum entropy method introduces the optimum vicinity parameter $(\epsilon)$, itself as a new recurrence quantifier that may be useful in the characterization of non-stationarity in time series. Although $\mathrm{N} \leq 4$ was sufficient for all tested systems, a possible drawback is expected for systems requiring $\mathrm{N}>4$ since the number of microstates grows substantially, which scales up the computational time.

We explore the maximum recurrence entropy $S_{\max }$ using the logistic map. In Ref. 17, the recurrence entropy (fixed $\epsilon$ ) is compared with the Lyapunov exponent. Here, we correlate $S_{\max }$ with the Lyapunov exponent and find even a better correlation than the previously obtained result with fixed $\epsilon .{ }^{17}$ Moreover, we have shown that $\mathrm{S}_{\max }$ displays a more detailed structure of the bifurcation diagram of the logistic map than any fixed $\epsilon$ recurrence entropy. Because of the detailed structure of the $S_{\max }$ and the better correlation between $S_{\max }$ and the Lyapunov exponent, we call $S_{\max }$ an optimal quantifier when compared with a fixed epsilon S. Reference 17 compares the recurrence microstate entropy with several other methodologies based on the recurrence plot showing similar or even better results. Here, we show that the maximum entropy concept improves considerably the fixed $\epsilon$ approach of Ref. 17 .

To conclude, we use entropy maximization to construct a proper index for the recurrence plot, and by consequence, to create an index that summarizes the information of the diversity of dynamical patterns represented in the recurrence plot. We remark that we are not adapting the function entropy as an index to characterize the diversity of a set of elements. In this work, we construct for the first time an entropy that attains a maximal value by adjusting automatically the vicinity parameter, and this quantity is used as a new index to measure the diversity of recurrence dynamical patterns. In addition, we have presented a new analysis of dynamic patterns that can be applied in future works to experimental signals, such as polysomnography, ${ }^{27,28}$ physiological signals, ${ }^{29-31}$ phase transition effect, ${ }^{32-34}$ turbulence process, ${ }^{35,36}$ and economic and geologic data. $^{37,38}$

\section{ACKNOWLEDGMENTS}

The authors acknowledge the support of Conselho Nacional de Desenvolvimento Científico e Tecnológico, CNPq-Brazil. G. Corso also gratefully acknowledges support from Shell Brasil through the project "New methods for Full Wave Inversion" at Programa de Pós Graduação em Ciências e Engenharia do Petróleo and the strategic importance of the support given by ANP through the R\&D levy regulation. We thank Jorge Lopez for the careful reading of the manuscript. We wish to acknowledge the support from São Paulo Research Foundation (Project No. FAPESP 2015/50122-0). This study was financed in part by the Coordenação de Aperfeiçoamento de Pessoal de Nível Superior-Brasil (CAPES)_Finance Code 001.

\section{REFERENCES}

${ }^{1}$ E. T. Jaynes, Phys. Rev. 106, 620 (1957).

${ }^{2}$ E. T. Jaynes, Phys. Rev. 108, 171 (1957).

${ }^{3}$ S. J. Phillips, R. P. Anderson, and R. E. Schapire, Ecol. Modell. 190, 231 (2006).

${ }^{4}$ A. Tang, D. Jackson, J. Hobbs, W. Chen, J. L. Smith, H. Patel, A. Prieto,

D. Petrusca, M. I. Grivich, A. Sher et al., J. Neurosci. 28, 505 (2008).

${ }^{5}$ F. Reif, Fundamentals of Statistical and Thermal Physics (McGraw-Hill, 1965).

${ }^{6}$ C. E. Shannon, ACM SIGMOBILE Mobile Comput. Commun. Rev. 5, 3 (2002).

${ }^{7}$ A. Hald, Stat. Sci. 14, 214 (1999).

${ }^{8}$ A. E. Magurran, Measuring Biological Diversity (Blackwell Science Ltd., 2004).

${ }^{9}$ J. Sueur, S. Pavoine, O. Hamerlynck, and S. Duvail, PLoS One 3, e4065 (2008).

${ }^{10}$ D. S. McNamara, M. M. Louwerse, P. M. McCarthy, and A. C. Graesser, Discourse Process. 47, 292 (2010).

${ }^{11}$ C. Bandt and B. Pompe, Phys. Rev. Lett. 88, 174102 (2002).

${ }^{12}$ H. Poincaré, Acta Math. 13, A3 (1890).

${ }^{13}$ C. L. Webber, Jr. and J. P. Zbilut, J. Appl. Physiol. 76, 965 (1994).

${ }^{14}$ N. Marwan, M. C. Romano, M. Thiel, and J. Kurths, Phys. Rep. 438, 237 (2007).

${ }^{15}$ C. Letellier, Phys. Rev. Lett. 96, 254102 (2006).

${ }^{16}$ P. beim Graben and A. Hutt, Philos. Trans. R. Soc. A 373, 20140089 (2015).

${ }^{17}$ G. Corso, T. L. Prado, G. Z. dos Santos Lima, J. Kurths, and S. R. Lopes, Chaos 28, 083108 (2018).

${ }^{18}$ M. Thiel, M. C. Romano, J. Kurths, R. Meucci, E. Allaria, and F. T. Arecchi, Physica D 171, 138 (2002).

${ }^{19}$ T. L. Prado, G. Z. dos Santos Lima, B. Lobão-Soares, G. C. do Nascimento, G. Corso, J. Fontenele-Araujo, J. Kurths, and S. R. Lopes, Chaos 28, 085703 (2018).

${ }^{20}$ S. R. Lopes, T. L. Prado, G. Corso, G. Z. dos Santos Lima, and J. Kurths, Chaos Solitons Fractals 133, 109616 (2020).

${ }^{21}$ J. P. Eckmann, S. O. Kamphorst, and D. Ruelle, Europhys. Lett. 4, 973 (1987).

${ }^{22}$ D. B. Vasconcelos, S. R. Lopes, R. L. Viana, and J. Kurths, Phys. Rev. E 73, 056207 (2006).

${ }^{23}$ T. L. Prado, P. P. Galuzio, S. R. Lopes, and R. L. Viana, Chaos 24, 013106 (2014). ${ }^{24}$ J. P. Zbilut and C. L. Webber, Jr., Phys. Lett. A 171, 199 (1992).

${ }^{25} \mathrm{M}$. Koebbe, G. Mayer-Kress, "Use of recurrence plots in the analysis of timeseries data," in Nonlinear Modeling and Forecasting, XXI, Proceedings of SFI Studies in the Science of Complexity, edited by M. Casdagli and S. Eubank (Addison-Wesley, Redwood City, 1992), pp. 361-378.

${ }^{26}$ K. T. Alligood, T. D. Sauer, and J. A. Yorke, Chaos (Springer, 1996).

${ }^{27}$ S. Redline, M. H. Sanders, B. Lind, S. F. Quan, C. Iber, D. J. Gottlieb, W. H. Bonekat, D. M. Rapoport, P. L. Smith, and J. P. Kiley, Sleep 21, 759 (1998).

${ }^{28}$ M. J. Mador, T. J. Kufel, U. J. Magalang, S. Rajesh, V. Watwe, and B. J. Grant, Chest 128, 2130 (2005).

${ }^{29}$ K. H. Kim, S. W. Bang, and S. R. Kim, Med. Biol. Eng. Comput. 42, 419 (2004).

${ }^{30}$ G. dos Santos Lima, B. Lobao-Soares, G. do Nascimento, A. S. Franca, L. Muratori, S. Ribeiro, and G. Corso, PLoS One 9, e105092 (2014).

${ }^{31}$ J. T. Cacioppo and L. G. Tassinary, Am. Psychol. 45, 16 (1990).

${ }^{32}$ G. dos Santos Lima, M. Corrêa, R. Sommer, and F. Bohn, Phys. Rev. E 86, 066117 (2012).

${ }^{33}$ E. Brézin and J. Zinn-Justin, Nucl. Phys. B 257, 867 (1985).

${ }^{34}$ G. Z. dos Santos Lima, S. R. Lopes, T. L. Prado, B. Lobao-Soares, G. C. do Nascimento, J. Fontenele-Araujo, and G. Corso, PLoS One 12, e0176761 (2017).

${ }^{35}$ M. T. Landahl, E. Mollo-Christensen, and M. S. Korman, Turbulence and Random Processes in Fluid Mechanics (Acoustical Society of America, 1989).

${ }^{36}$ P. Galuzio, S. Lopes, G. dos Santos Lima, R. Viana, and M. Benkadda, Physica A 402, 8 (2014).

${ }^{37}$ D. Acemoglu, J. Econ. Theory 147, 545 (2012).

${ }^{38}$ B. L. Connelly, S. T. Certo, R. D. Ireland, and C. R. Reutzel, J. Manage. 37, 39 (2011). 